\title{
MAPEAMENTO DAS PUBLICAÇÕES CIENTÍFICAS SOBRE A LIDERANÇA EM ORGANIZAÇÕES INTENSIVAS EM CONHECIMENTO
}

Fabiana Besen SANTOS ${ }^{1}$

Edivandro TECCHIO ${ }^{2}$

Francisco Antônio Pereira FIALHO ${ }^{3}$

\author{
${ }^{1}$ Doutoranda do Programa de Engenharia e Gestão do Conhecimento - UFSC. fabibesen@ gmail.com \\ ${ }^{2}$ Doutorando do Programa de Engenharia e Gestão do Conhecimento - UFSC. edivandro@gmail.com \\ ${ }^{3}$ Doutor, professor no Programa de Engenharia e Gestão do Conhecimento - UFSC. fialho@egc.ufsc.br
}

Recebido em: 24/05/2014 - Aprovado em: 30/06/2014 - Disponibilizado em: 30/07/2014

\begin{abstract}
Resumo: A liderança em organizações intensivas em conhecimento é entendida como um processo de suporte e inspiração para os trabalhadores do conhecimento, contribuindo para o bom desempenho organizacional. O objetivo deste artigo é apresentar um mapeamento das publicações científicas sobre liderança em organizações intensivas em conhecimento. Para isso, foi utilizada a bibliometria como uma reconhecida técnica de medição da produção e disseminação do conhecimento científico. Os resultados demonstram que no Brasil, até o presente momento, o tema não foi objeto de estudo dos pesquisadores; em nível internacional, ainda é pouco investigado. Constatou-se também que os estudos sobre a liderança em organizações intensivas em conhecimento tem foco na identificação dos desafios da liderança, das características e habilidades dos líderes e no papel dos líderes no processo de gestão do conhecimento.
\end{abstract}

Palavras-chave: Liderança; Organizações; Conhecimento; Bibliometria; Gestão do Conhecimento.

\section{MAPPING OF SCIENTIFIC PUBLICATIONS ABOUT LEADERSHIP IN KNOWLEDGE INTENSIVE ORGANIZATIONS}

Summary: Leadership in knowledge intensive organizations is understood as a process of support and inspiration for knowledge workers, contributing to good organizational performance. The objective of this paper is to present a mapping of scientific publications on leadership in knowledge intensive organizations. For this, we used bibliometrics as a recognized technique for measuring the production and dissemination of scientific knowledge. The results show that in Brazil, until now, the subject was not the object of study from researchers; internationally, is still poorly investigated. It was also found that studies of leadership in knowledge intensive organizations have focused on identifying the challenges of leadership, characteristics and skills of the leaders and the role of leaders in the knowledge management process.

Keywords: Leadership; Organizations; Knowledge ; Bibliometrics ; Knowledge Management. 


\section{Introdução}

Nossa sociedade vivencia um período de transformação, que se caracteriza por um processo de reorganização e reformulação de sua visão de mundo, dos seus valores básicos e de suas estruturas sociais e políticas. As atividades que ocupavam o lugar central das organizações deixam de ser aquelas que visam produzir ou distribuir objetos e passam a ser aquelas que produzem e distribuem informação e conhecimento (DRUCKER, 1993). São as chamadas organizações intensivas em conhecimento (OIC), definidas como organizações que oferecem ao mercado produtos baseados no conhecimento (ALVESSON, 2004), ou seja, o conhecimento é o seu principal input de produção (STARBUCK, 1992). Em razão dessas características, esse tipo organizacional precisa ter a capacidade de aprendizagem constante a fim de gerar valor e inovação.

Neste ambiente, a gestão também precisa acompanhar essas mudanças para que mantenham sua vantagem competitiva (MAXIMIANO, 2004). A condução dos novos trabalhadores do conhecimento na direção da eficiência e eficácia exigem novas características e habilidades. Assim, a compreensão da liderança no contexto das organizações intensivas em conhecimento passa a ter um papel preponderante, visto que cabe ao líder influenciar os subordinados a adotar um comportamento inovador.
A partir deste cenário, este artigo buscou mapear as publicações científicas sobre a liderança em organizações intensivas em conhecimento, contribuindo com a identificação das características dos trabalhos, como autores que contribuem para o tema, período das publicações, instituições e países onde o tema é estudado, periódicos nos quais os trabalhos são publicados, entre outros. Para isso foram utilizadas técnicas bibliométricas, que empregam métodos quantitativos na busca por uma avaliação objetiva da produção científica.

\section{Liderança e Organizações Intensivas em conhecimento}

Os estudos sobre gestão enfatizam a importância da liderança para o alcance dos resultados organizacionais (BARNEY, 1996; BASS, 1990). A liderança geralmente envolve visões, cooperação, trabalho em rede, trabalho em equipe, criatividade e inspiração (YULK, 2005; KOTTER, 1990; NORTHOUSE, 2004). O líder é considerado uma fonte de inspiração e suporte (KOTTER, 1990), que visa à congregação de esforços, com o fim de alcançar uma visão comum. De forma consensual, a liderança deve levar em consideração o contexto, a cultura e a estrutura organizacional (ALVESSON; SVENINGSSON, 2003).

O cenário das organizações intensivas em conhecimento é peculiar. Uma empresa do 
conhecimento é caracterizada por criar valor por meio do uso do conhecimento (ALVESSON, 2004). Assim, o conhecimento é o seu principal input para desenvolvimento de seus produtos (STARBUCK, 1992), diferente das empresas de produção que utilizam o trabalho. Nas OICs, a maior parte do trabalho é intelectual. Isso quer dizer que se baseia na capacidade cognitiva ao invés da força física. No ambiente das OICs, são as competências das pessoas que impulsionam o desenvolvimento dos produtos (ALVESSON; SVENINGSSON, 2003), por isso para muitos autores (ALVESSON; SVENINGSSON, 2003; ALVESSON, 2004; STARBUCK, 1992) o conhecimento dos membros da organização é um importante fator de sucesso dessas empresas.

Nos estudos encontrados sobre a liderança em organizações intensivas em conhecimento, foram identificados três temas importantes que serão tratados neste artigo: os desafios da liderança em OICs (ALVESSON; SVENINGSSON, 2003a; ALVESSON; SVENINGSSON, 2011); as características e habilidades dos líderes em OICs (ALVESSON; SVENINGSSON, 2011; ALVESSON; SVENINGSSON, 2003b) e o papel do líder na gestão do conhecimento em OICs (JONG; HARTOG, 2007).

\section{Método}

Este trabalho tem natureza exploratória de caráter descritivo. A busca sistemática da literatura foi desenvolvida a partir da aplicação de procedimentos e técnicas oriundos da bibliometria (BUFREM; PRATES, 2005). A bibliometria é uma técnica que emprega métricas quantitativas dos registros bibliográficos, por meio das quais é possível medir a produção científica, o perfil e a disseminação das pesquisas em um determinado campo de conhecimento (ARAUJO, 2006).

Para realizar este trabalho, foram desenvolvidas duas etapas: 1) coleta de dados; e 2) representação dos dados e análise dos dados. Nas subseções 3.1 e 3.2 explicam a etapa de coleta de dados (etapa 1) para os estudos em nível nacional e internacional, respectivamente. A subseção 3.3 descreve as representações e análise dos dados (esses resultados estão apresentados na seção 4 deste artigo.

\subsection{Coleta de dados para a bibliometria nacional}

De forma a obter o mapeamento das publicações nacionais, foram utilizados como fontes de dados periódicos nacionais listados no Web Qualis (CAPES). Neste trabalho optou-se por utilizar os periódicos mais bem posicionados nessa classificação. Dessa forma, foram selecionados os periódicos classificados como A1, A2 e B1 das áreas Administração e Interdisciplinar, contendo no título pelo menos uma das palavras: Gestão, 
Organizacão(ões), Administração (ou em inglês Administration) e Ciências Sociais.

As buscas nos periódicos nacionais foram realizadas utilizando-se a expressão ("liderança” AND “organizações intensivas em conhecimento") OR ("liderança” AND "pequenas e médias empresas intensivas em conhecimento") OR ("liderança" AND “organizações baseadas em conhecimento”) OR ("liderança” AND "firmas intensivas em conhecimento") OR ("liderança” AND “organizações baseada em informação”). Cada periódico acima mencionado foi acessado individualmente, aplicando-se a expressão de busca nos campos disponíveis do site consultado. Uma vez que não existe padronização nos campos de busca entre os diferentes periódicos, para todos os periódicos foi utilizado o campo de busca que corresponde a todos os índices disponíveis (resumo, palavras do título, autor, texto completo, entre outros).

$\mathrm{O}$ período aplicado às buscas nacionais correspondeu ao tempo de existência de cada periódico, ou seja, as buscas foram realizadas de forma a abranger todas as publicações de todos os periódicos, sem delimitações de período.

\subsection{Coleta de dados para bibliotmetria} internacional

Para realizar o estudo bibliométrico em nível internacional foram utilizadas três bases de dados que indexam publicações científicas: a Web of Science (WoS), a Scopus e a $E S B C O$ consideradas pela comunidade científica como relevantes fontes para estudos bibliométricos.

$\mathrm{Na} \mathrm{WoS}$ foram selecionadas todas as sub-bases e foi utilizado o período de busca disponível na base até o último ano incompleto: 1945-2013 (dezembro). $\mathrm{Na}$ Scopus, foram utilizadas todas as a sub-bases disponíveis e o período aplicado também foi o disponível na base até o último ano incompleto: 1960-2013 (dezembro). Na Ebsco, também foram selecionadas todas as sub-bases e utilizado o período de busca disponível até o último ano incompleto (outubro de 2013). A escolha das sub-bases citadas é justificada pelo fato de que o tema liderança ser multidisciplinar, sendo estudado por diversas áreas.

Com a finalidade de realizar uma ampla cobertura das publicações sobre o tema liderança em organizações intensivas em conhecimento, foi investigada a área como um todo por meio da combinação de várias estratégias de buscas. Dessa forma, as buscas foram realizadas utilizando-se as expressões: "leader*" AND "information-based organi?ation"; "leader*"AND "knowledgeintensive small AND medium enterprise"; leader" AND "knowledge-intensive organi?ation"; "leader" "AND "knowledgebased organi?ation"; "leader*" AND "knowledge-intensive firm", nos campos de busca TOPIC (título, palavras-chave e 
resumos) na Web of Science, no campo de busca correspondente a "título, palavraschave, resumo", na base Scopus e no campo de busca título, palavras-chave e resumo na base Ebsco. O símbolo “?” representa a busca por qualquer caractere nessa posição (permite recuperar os termos nas variações entre o inglês britânico - organisational, e o americano - organizational). O uso do caracter “*” no termo "leader*" permitiu buscar tanto os substantivos "líder" e "liderança".

\subsection{Representação e análise dos dados}

As análises dos dados foram feitas a partir da representação das informações bibliométricas. Para gerar essas representações, as informações das publicações localizadas nas três bases de dados internacionais foram exportadas para os EndNote e HistCite. Esses softwares possibilitam a organização e visualização dos dados bibliográficos provenientes de bases que indexam publicações, permitindo uma análise dos dados mais completa. Com o apoio dessas ferramentas foram gerados gráficos, as tabelas e imagens para representar as seguintes informações bibliométricas: frequência das publicações por anos; os periódicos com mais publicação sobre o tema; os principais autores, instituições e países; palavras-chave mais utilizadas e os trabalhos mais citados.
A síntese dos resultados obtidos a partir do desenvolvimento de cada etapa de pesquisa, apresentada anteriormente, é apresentada na próxima seção deste artigo.

\section{Resultados}

\subsection{Representação e análise dos dados nacionais}

As buscas realizadas nos periódicos nacionais não retornaram registros de publicações científicas sobre liderança em organizações intensivas em conhecimento. Dessa forma, pode-se considerar que o tema não é objeto de estudo da comunidade científica brasileira, nos periódicos selecionados para consulta.

\subsection{Dados internacionais}

Para a bibliometria das publicações sobre liderança em organizações intensivas em conhecimento, em nível internacional, as buscas realizadas retornaram 9 trabalhos acadêmicos indexados à Web of Science, 23 trabalhos indexados à Scopus e 6 trabalhos indexados à Ebsco, totalizando 38 publicações. Desse total, 13 trabalhos repetiram-se entre as bases, sendo então eliminados, resultando um conjunto de 25 trabalhos para a análise da literatura.

Os 25 trabalhos foram escritos por 47 autores vinculados a 20 instituições de 12 países diferentes e estão publicados em 24 periódicos/ eventos.

A Figura 1 apresenta a distribuição temporal dos 25 trabalhos identificados. 
Percebe-se que, o estudo da liderança em organizações intensivas em conhecimento é recente, iniciado em 1997, ou seja, há 16 anos, a maior parte das publicações, representada por $68 \%$, ocorreu a partir de 2006. Identifica-se que o tema em estudo recebeu maior atenção nos últimos 8 anos.

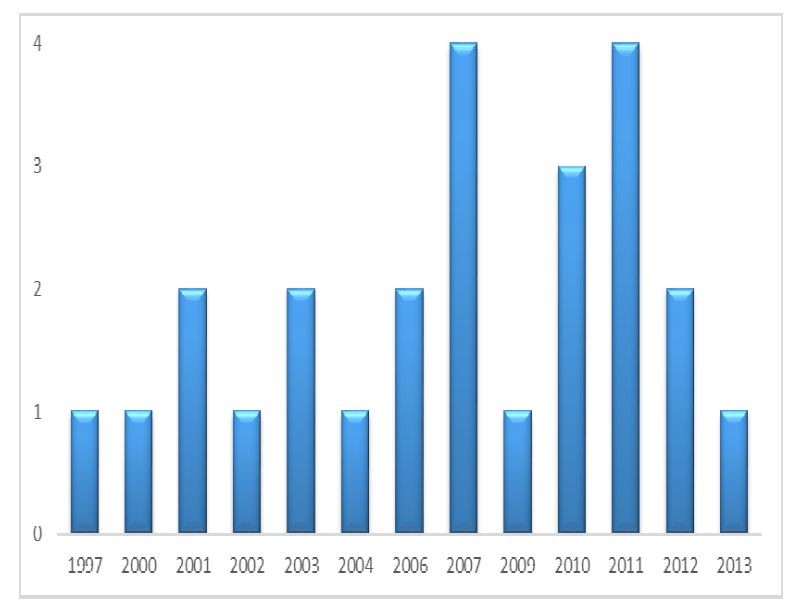

Figura 1. Frequência das publicações por ano no período (1997-2013).

$\mathrm{Na}$ sequência, foram analisados os periódicos com maiores frequências de artigos publicados sobre o tema. Observou-se que não tem um período ou evento de destaque com publicações sobre o tema, apenas o European Journal of Innovation Management que tem 2 publicações, uma a mais que os demais 22 períodos e eventos.

Quanto aos países de origem das publicações, os Estados Unidos lidera a lista com uma frequência de 9 artigos publicados, representando $36 \%$ da quantidade total, seguido pelo Reino Unido com 4 artigos, 16\% da quantidade total e Suécia com 3 artigos, 12 $\%$ do total. Observa-se uma concentração entre esses três países, pois são responsáveis por $64 \%$ das publicações sobre o tema enquanto que os $36 \%$ restantes estão distribuídos entre 9 países.

Dentre as 20 instituições que estudam sobre a liderança em organizações intensivas em conhecimento, observou-se que o maior número de publicações está distribuído em quatro instituições. Embora os Estados Unidos sejam o país que mais publica sobre o tema, a lista das instituições é liderada Lund University, localizada na Suécia.

Dentre os autores com maior número de trabalhos publicados sobre a liderança em organizações intensivas em conhecimento, destaca-se Mats Alvesson e Stefan Sveningsson, com 3 publicações realizadas em conjunto. Em seguida vem os autores Holsaplle e Joshi, com 2 trabalhos também em conjunto. O restante dos autores tem apenas 1 publicação cada um.

A Figura 2 mostra as palavras-chave mais utilizadas representadas de acordo com sua frequência. As 7 palavras-chave mais utilizadas com sua respectiva frequência são: leadership (12), knowledge (10), knowledge management (8) innovation (7), societies $e$ institutions (7), organizations knowledge (5) e knowledge sharing (4). A palavra chave leadership, que reflete exatamente um dos temas principais desta pesquisa, aparece na $1^{\mathrm{a}}$ posição com 12 repetições. Vale ressaltar que a palavra-chave knowledge ganha destaque na nuvem de palavras, pois participa de várias outras palavras-chave compostas, como 
knowledge transfer, knowledge learnin;

knowlegde information.

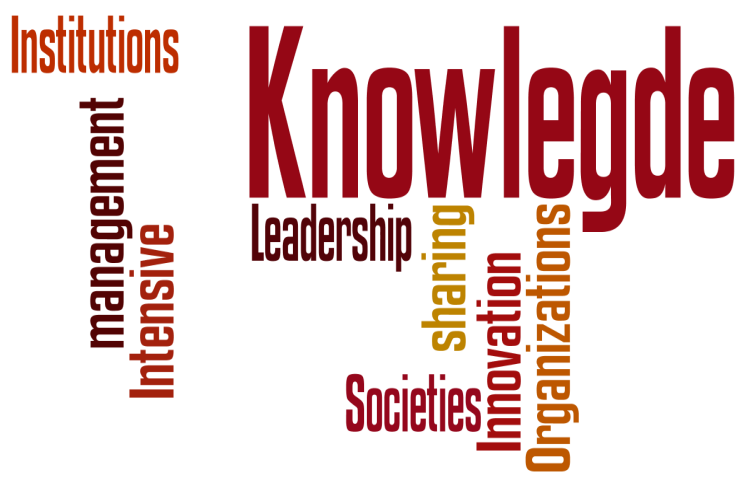

Figura 2: Representação das frequências das palavraschave.

Por fim, analisou-se a frequência de citações dos estudos sobre a liderança em organizações intensivas em conhecimento, de acordo com o indicador bibliométrico denominado de GCS (Global Citation ScoreEscore Global de Citações) que se refere à quantidade de vezes que os trabalhos foram citados por outros trabalhos nas bases WoS, Scopus e Ebsco. Observa-se que os três principais trabalhos são intitulados: Managers doing leadership: The extra-ordinarization of the mundane com 47 citações, How leaders influence employees' innovative behaviour, com 44 citações e Good Visions, Bad Micromanagement and ugly ambiguity: contradictions of (non-) leadership in a knowledge-intensive organization, com 43 citações. Somados os três artigos têm 134 citações, representando $56 \%$ das citações entre os artigos mais citados.

\subsection{Temas relacionados à liderança em organizações intensivas em conhecimento}

Dentre os 25 artigos identificados, foram observados os principais temas que os autores relacionados com a liderança em organizações intensivas em conhecimento, como: desafios da liderança, características e habilidades no processo da liderança e a liderança na gestão do conhecimento.

Desafios da liderança: A realidade das organizações intensivas em conhecimento é de ambiguidade e complexidade (ALVESSON; SVENINGSSON, 2003a). Um fator que reforça o cenário de ambiguidade e complexidade é a dificuldade de avaliação e expectativas de progressão de tarefas no trabalho, por exemplo, desenvolvimento de produtos, é complicado especificar os detalhes exatos e pode, subsequentemente, ser interpretada de diferentes maneiras (ALVESSON, SVENINGSSON, 2011). Essas condições ambíguas acabam levando a contextos mais criativos e inovadores, por isso muitos autores associam ambiguidade com a inovação (ALVESSON, 2004).

\section{Características e habilidades do}

líder: O líder no ambiente das empresas do conhecimento é um fator significativo na influência do comportamento de aprendizagem e inovação dos trabalhadores (KIM，NEWBY-BENNETT，SONG，2012; JONG E HARTOG, 2007). É entendida como um suporte e uma inspiração (ALVESSON, SVENINGSSON, 2003a), a formulação da 
visão central e a condução para o alcance da mesma (KOTTER, 1990; FAGIANO, 1997). A comunicação aparece como um tema central na liderança nas organizações intensivas em conhecimento (ALVESSON, SVENINGSSON, 2011, KIM, NEWBYBENNETT, SONG, 2012). Escutar é uma habilidade do líder necessária em determinadas circunstâncias para uma liderança eficaz (WRIGHT, TAYLOR, 1994). Ouvir pode fazer os subordinados se sentirem mais favoráveis aos gestores no momento das discussões de problemas e também pode resultar em menor resistência à mudança (ALVESSON, SVENINGSSON, 2011).

\section{Papel do líder na gestão do} conhecimento: Os líderes de organizações intensivas em conhecimento desempenham um papel importante como facilitadores da gestão do conhecimento (JONG E HARTOG, 2007). Podem atuar como facilitadores de compartilhamento de conhecimento e parceiros na tomada de decisão. A liderança no processo de gestão do conhecimento afeta também a criação de conhecimento. Os líderes nesses tipos de empresas precisam estabelecer uma cultura e um design organizacional que se reforçam mutuamente (LINDERMAN, SCHROEDER, SANDERS, 2010).

Observa-se que a liderança no contexto das organizações intensivas em conhecimento é tipicamente visionária criativa, inspiradora, inovadora e corajosa
(ALVESSON, SVENINGSSON 2003a), sendo importante no processo de aprendizagem e adaptação às mudanças ambientais, contribuindo com a manutenção da competitividade e o desempenho da empresa.

\section{Considerações Finais}

Este artigo se propôs a realizar um mapeamento quantitativo das publicações científicas sobre o tema liderança em organizações intensivas em conhecimento. No Brasil, constatou-se que, até o presente momento, o tema não foi objeto de estudo dos pesquisadores, considerando as publicações dos periódicos selecionados das áreas de Administração e Interdisciplinar (Qualis/Capes), anteriormente referenciados. Em nível internacional, o tema ainda é pouco investigado, despertando maior atenção da academia a partir de 2006. Os estudos são provenientes de pesquisadores concentrados nos Estados Unidos, Reino Unido e Suécia, sendo os autores e instituições desses países que mais contribuem para o tema, com as maiores quantidades de trabalhos publicados.

Nos artigos analisados, detectou-se que a liderança em organizações intensivas em conhecimento é peculiar em razão do contexto onde essas organizações estão inseridas. Entre seus principais desafios estão a ambiguidade e complexidade do ambiente dessas organizações e o perfil dos subordinados, que exigem autonomia, liberdade e independência, dificultando o 
monitoramento das tarefas por parte do líder.

Os líderes nas empresas do conhecimento apresenta características e habilidades específicas que enfatizam a importância da comunicação. Os líderes são vistos como facilitadores no processo de gestão do conhecimento, em especial nas etapas de criação e compartilhamento do conhecimento.

Espera-se que os resultados apresentados nesse artigo colaborem para a disseminação e conscientização da importância da liderança nas organizações intensivas em conhecimento e contribua para o crescimento das pesquisas sobre o tema, em especial no Brasil, onde não existem publicações nos periódicos pesquisados.

\section{Referências}

ALVESSON, M. Knowledge work and knowledge-intensive firms. Oxford: Oxford University Press, 2004.

Knowledge work. Ambiguity, image and identity. Human Relations, v. 54, n. 7, p. 863-886, 2001.

Management of knowledge-intensive companies. Berlin: De Gruyter, 1995.

ALVESSON, M.; SVENINGSSON, S. Management is the solution: Now what was the problem? On the fragile basis for managerialism. Scandinavian Journal of Management, v. 27, p. 349-361, 2011.

Good Visions, Bad Micro-management and ugly ambiguity: contradictions of (non-) leadership in a knowledge-intensive organization. Organization Studies: London, v. 24, n. 6, p 961-988, 2003a.
Managers doing leadership: The extraordinarization of the mundane. Human Relations, v. 56, n. 12, p 1435-1459, 2003 b.

ARAUJO, C. A. Bibliometria: evolução, história e questões atuais. Em Questão, v. 12, n. 1, p. 11-32, 2006.

BARNEY, J. Bringing managers back in. In A. Malm (Ed.) Does management matter? Institute of Economic Research Crafoord Lectures $6 . \quad$ Lund: Lund University Press, 1996.

BASS, B. M. Bass of stogdill's handbook of leadership: theory, research and managerial application, New York, The Free Pres, 1990.

BRYMAN, A., STEPHENS, M., CAMPO, C. The importance of context: Qualitative research and the study of leadership. Leadership Quarterly, v. 7, n. 3, p. 353-370, 1996.

BUFREM, L.; PRATES, Y. O saber científico registrado e as práticas de mensuração da informação. Ciência da Informação, v. 34, n. 2, p. 9-25, 2005.

DRUCKER, P. F. Sociedade pós capitalista. 2 ed. São Paulo, Pioneira, 1994.

EDMONDSON, E. Speaking up in the operating room: how team leaders promote learning in interdisciplinary action teams. Journal of Management Studies, v. 40, n. 6, p. 1419-1452, 2003.

FAGIANO, D. Managers vs. leaders: A corporate fable. Management Review, v. 10, n. 5, 1997.

JONG, J. P. J. de; HARTOG, D. N. D. How leaders influence employees' innovative behaviour, European Journal of Innovation Management, v. 10, n. 1, p. 41 - 64, 2007.

KIM, Y.-M.; NEWBY-BENNETT, D; SONG, H.-J. ,Knowledge sharing and institutionalism in the healthcare industry. Journal of Knowledge Management, v. 16, n. 3, p. $480-494,2012$. 
KOTTER, J. Force for change: How leadership differs from management. New York: Free Press, 1990.

KUIVALAINEN， O; SUNDQVIST， S.; PUUMALAINEN, K. The Effect of Environmental Turbulence and Leader Characteristics on International Performance: Are Knowledae-Based Firms Different? Canadian Journal of Administrative Sciences, p. 35-50, 2004.

LINDERMAN， K.; SCHROEDER，R. G.; SANDERS, J. A Knowledge Framework Underlying Process Management. Decision Sciences, v. 41, n. 4, 2010.

MAXIMIANO, A. A. Introdução a Administração. 6 ed. São Paulo: Atlas, 2004.

MCKENNA, B; ROONEY, D; BOAL, K. B. Wisdom principles as a meta-theoretical basis for evaluating leadership. The Leadership Quarterly, v. 20, p. 177-190, 2009.

MINTZBERG, H.; MCHUGH, A. Strategy formation in an adhoc-racy. Administrative Science Quarterly, v. 30, p. 160-197, 1985.

MINTZBERG, H. Covert leadership: Notes on managing professionals. Harvard Business Review, p. 140-147, nov./ dez. 1998.

NORTHOUSE, P. G. Leadership: Theory and practise. Sage Publications, Inc.:3 ed. 2004.

STARBUCK, W. Learning by knowledgeintensive firms. Journal of Management Studies, v. 29, p. 713-740, 1992.

VICENZI, R.; ADKINS, G. A Tool for Assessing Organizational Vitality in an Era of Complexity. Technological Forecasting and Social Change, v 64, n. 1, p.101-113, 2000.

WRIGHT, P.; TAYLOR, D. Improving leadership performance, 2 ed. Hemel Hempstead: Prentice Hall, 1994.

YUKL, G. Leadership in Organization. 6 ed. Upper Saddler River, NJ: Pearson Prentice Hall, 2005. 